\title{
CO-CONSTRUCTION DE SENS EN SITUATION DE CONCEPTION D'UN OUTIL DIDACTIQUE
}

\begin{abstract}
A bstract. Brassac Christian, Grégori Nicolas, Co-construction de sens en situation de conception d'un outil didactique [Common construction of sense during creation of a certain type of didactic aids]. Studia Romanica Posnaniensia. Adam Mickiewicz University Press, Poznań, vol. XXV/XXVI: 2000, pp. 55-66. ISBN 83-232-0965-0. ISSN 0137-2475.

In the article, the author defends a thesis on the necessity of application in psychology and other cognitive sciences of a methodology based on the interactive theory. Therefore, it is postulated that analysis from the perspective of a monologue be replaced by the analysis from the interactive perspective. The article consists of two parts. In the first part a model of mutual understanding is presented, which was devised at the Psychological Laboratory of the Nancy University 2, and in the other, a proposal of application of this model for the developing of didactic aids of a multimedial character is discussed.
\end{abstract}

\section{INTRODUCTION}

C'est en tant que psychologues que nous contribuons à cette rencontre centrée autour de l'analyse de discours. Psychologues de l'interaction qui nous intéressons aux processus cognitifs en situation intersubjective et pour qui l'interaction conversationnelle est le prototype de la pratique discursive que les sujets humains mettent en oeuvre quotidiennement. En interagissant au moyen de l'usage du langage en situation, les agents conversant génèrent cognitions conjointes et rapports sociaux. $\mathrm{Ce}$ faisant, ils co-construisent le sens des formes langagières qu'ils produisent, ils modifient en profondeur et de façon continue les conditions contextuelles d'énonciation qui les rassemblent, ils contribuent à l'élaboration de la réalité sociale. On reconnaît dans cette suite d'affirmations, une reformulation de ce qui constitue les propriétés de l'ethnométhode par excellence qu'est la conversation.

De fait, la méthodologie d'analyse de discours interlocutoire, que nous allons succinctement évoquer d'une part et dont nous allons montrer la pertinence pour un do- 
maine d'études particulier d'autre part, relève d'un paradigme conversationnaliste même si elle n'en partage pas totalement les outils d'analyse. Nous défendrons en effet dans cet article l'inscription en analyse conversationnelle (la conversation y sera alors abordée en tant qu'elle est à la fois productrice de rapports sociaux et de processus cognitifs) tout en prônant l'usage méthodologique de la catégorie 'acte de langage' (plus précisément, c'est une version dialogisée de la sémantique générale, dernière axiomatisation de la théorie standard des actes de langage, qui nous servira de dispositif d'analyse des corpus conversationnels).

Plus généralement, il s'agit pour nous de plaider pour l'adoption en psychologie, et plus généralement en sciences cognitives, d'une démarche radicalement interactionniste. L'appel au principe interactionniste est certes souvent effectué, mais rarement étayé par une méthode d'analyse solide. Nous voulons fonder ce recours à l'interaction sur une méthodologie d'analyse des phénomènes cognitifs qui donne le primat à la relation inter-entités porteuses de mécanismes cognitifs et qui, ce faisant, abandonne une démarche monologique au profit d'une perspective dialogique.

Dans un premier temps, nous exposerons le modèle de l'intercompréhension que nous avons contribué à élaborer au sein du Laboratoire de Psychologie de l'Interaction de l'Université Nancy 2. Dans un second temps, nous montrerons en quoi, nous pouvons le mettre à l'épreuve dans le cadre de la conception, que l'on dira située et distribuée, d'un outil pédagogique multimédia.

\section{L'INTERCOMPRÉHENSION COMME MOTEUR DE CONSTRUCTION DE COGNITIONS DISTRIBUÉES ET SITUÉES}

\subsection{L'ACTE DE LANGAGE, UNITÉ D'ANALYSE}

Le point d'entrée dans la problématique relève de l'analyse des conversations. Le postulat est le suivant: il est possible d' «atteindre» les processus cognitifs humains en analysant les productions langagières des sujets en situation interlocutoire. Mieux, une description fine de l'enchanîement conversationnel, à fin modélisatrice, est une méthode fiable de mise à jour des mécanismes de la cognition humaine. Nous agissons donc en tant que théoricien de l'interaction sociale en l'envisageant au plan micro. Bien sûr il n'est pas question de nier l'importance des aspects paraverbaux et non verbaux (notre méthodologie passe par un recueil de données vidéo), mais nous prenons le parti de nous intéresser à l'usage du langage par les sujets humains en situation interactionnelle car un ensemble de conceptions de cet usage permet d'en envisager et la fonction représentationnelle et la fonction intentionnelle et actionnelle, de façon solidement raisonnée et théoriquement établie'. En effet, la théorisation

${ }^{1}$ Nous appelons de nos voeux la collaboration avec des chercheurs analysant finement par exemple la gestuelle, la prosodie ou autres questions relevant non directement des aspects verbaux de l'interaction. 
conjointe du phraséologique et du praxéologique est tout à fait fondamentale lorsqu'il s'agit de modéliser l'interaction... car si la première composante du mot, «inter», renvoie à la réciprocité, la seconde marque l'inscription de toute étude de cette relation dans le paradigme de la théorie de l'action. Ancrés en pragmatique linguistique, nous faisant nôtre la formule «user du langage c'est agir dans et sur le monde»; c'est dire que nous adoptons une vision radicalement actionnelle.

Comme nous le disions en introduction, c'est la notion d'acte de langage, en tout cas primitivement, qui nous sert de point de départ. Lorsque le sujet, en contexte (et plus précisément en contexte conversationnel), profère un énoncé, il réalise, il accomplit, par cette simple profération, un acte dit de langage. Cet acte est le maillon élémentaire qui forme la trame de l'enchainement conversationnel. La théorie des actes de langage aura abouti, dans un premier temps, à son axiomatisation en une logique illocutoire (Searle et Vanderveken, 1985), puis dans un second temps à l'élaboration d'une sémantique formelle générale (Vanderveken, 1988)2 ${ }^{2}$. Certes cette théorie est et a été vivement critiquée de toutes parts. Un, parce qu'elle est empreinte d'un monologisme radical et, deux, parce qu'elle est souvent considérée comme tout à fait inadéquate dans le rôle d'unité d'analyse de l'interaction verbale qu'elle fait jouer à l'acte de langage. Nous ne pourrons ici développer ces débats (pour plus de détails voir Brassac, 1992, 1944a, 1994b; Trognon et Brassac, 1992, 1995) mais il est clair qu'une des principales critiques est que cet appareillage formel qui modélise tout à fait complètement, via l'étude approfondie de l'acte de langage, l'expression et la compréhension du langage par des sujets humains, faillit gravement à rendre compte de l'usage du langage en situation de dialogue. À cela deux raisons principales: la non prise en compte des aspects non littéraux, tout à fait fondamentaux dans l'intersubjectivité, et le caractère statique d'une analyse qui n'aborde pas du tout le processus se jouant dans le déroulement conversationnel.

\subsection{DIALOGISME ET INTERSUBJECTIVITÉ}

Car l'enjeu est bien là pour qui veut tirer profit de cette théorie pour modéliser l'interaction dans son déploiement, pour qui veut rendre compte du processus interactionnel. La réponse consiste en ce que l'on pourrait appeler une «dialogisation» de cette sémantique formelle générale. Dialogisation dont l'objectif est de considérer le dialogisme ( $v s$ monologisme) comme non seulement central mais aussi comme devant être conceptualisé; dialogisation qui doit conduire à circonscrire et la gestion de la non littéralité et la dynamique de l'échange interlocutoire.

${ }^{2}$ Vanderveken réserve quant à lui l'usage du terme «pragmatique» à l'étude des aspects non littéraux, implicites de l'usage du langage, arguant du fait qu'il est nécessaire de construire cette sémantique générale (incluant la logique illocutoire sus-mentionnée) en restant en deçà de la frontiêre de la littéralité. 
Un analyste utilisant la théorie des actes de langage dans les deux versions citées ci-dessus est contraint de qualifier l'acte réalisé par la profération de l'énoncé (1) comme une assertion.

(1) Tu as le téléphone ici

En effet, il n'est pas niable que ce faisant le locuteur rend compte d'un état du monde, c'est-à-dire ajuste les mots au monde, et par conséquent réalise un acte assertif. Il est cependant difficile pour des conversants experts que nous sommes tous de ne pas pouvoir y voir une demande implicite d'information, voire d'action. Nous prétendons que la seule «vérité» en la matière est ce qui se passe effectivement dans l'ici et maintenant de la conversation au sein de laquelle il a été produit. Examinons ceci de façon plus précise sur cet ensemble d'exemples.

Une des quatre séquences suivantes, mettant en scène un homme (E) raccompagnant en voiture chez elle une jolie jeune fermme (L) et arrivant au pied de l'immeuble, a été observée et retranscrite.

séquence 1

$E_{1} \quad$ Tu as le téléphone ici

$\mathrm{L}_{\mathrm{l}} \quad$ Oui, c'est moderne

$\mathrm{E}_{2} \quad \mathrm{Ah} \ldots$ je n'aurais pas cru

séquence 2

$\mathrm{E}_{1} \quad$ Tu as le téléphone ici

$\mathrm{L}_{1} \quad$ Oui, c'est le numéro 04-83-35-36-09

$\mathrm{E}_{2} \quad$ Ah ben je pourrai(s) t'appeler comme ça

séquence 3

$\mathrm{E}_{1} \quad$ Tu as le téléphone ici

$\mathrm{L}_{1} \quad$ Oui, c'est moderne

$\mathrm{E}_{2}$ (rires) Ah ben j'aurais bien aimé que tu me donnes le numéro

séquence 4

$\mathrm{E}_{1} \quad$ Tu as le téléphone ici

$\mathrm{L}_{\mathrm{I}} \quad$ Oui, c'est le numéro 04-83-35-36-09

$\mathrm{E}_{2} \quad$ Euh, mais je ne te demandais pas le numéro

D'abord une question brutale; dans l'ensemble de ces quatre séquences, y en a-t-il une, ou plus, où les deux interactants se sont compris? Si l'on est tenté de répondre «oui, il se sont compris à la séquence 2» c'est que l'on pense que $\mathrm{E}$ avait pour intention communicative de demander le numéro de téléphone (et non pas seulement de demander si $\mathrm{L}$ avait le téléphone). Et par conséquent, que la séquence $4 \mathrm{n}$ 'est pas possible. Mais de quel droit peut-on affirmer ceci? Quel analyste a le pouvoir de décider que $\mathrm{E}$ demandait, implicitement le numéro? Remarquons qu'il n'est pas plus justifié de dire que E voulait, littéralement, savoir si $\mathrm{L}$ avait le téléphone ou non. Mais là n'est pas l'important. L'important c'est le sens que ces deux interactants donnent, dans l'ici et maintenant de la conversation, à $E_{1}$.

Plus techniquement, on peut dire que $\mathrm{E}_{1}$ est une assertion formalisable par ASS(p) et paraphrasable performativement par «Je dis que tu as le téléphone ici». Au titre des actes non littéraux associés à cette assertion, on peut évoquer sans doute une requête d'information formalisable par DIR(p) et paraphrasable performativement par «Je te demande si tu as le téléphone» ou aussi une requête d'action formalisable par DIR'(p') et paraphrasable performativement par «Je te demande ton numéro de téléphone». Il est possible que cet énoncé porte d'autres actes illocutoires (pourquoi pas une expression d'étonnement, d'admiration ou autres...?). Le fait décisif, c'est que $L$, en répliquant, satisfait, au sens technique du terme, soit DIR(p) (pour les séquences 1 et 3 ) soit DIR'(p') (pour les séquences 2 et 4 ). Ce faisant, selon une loi fondamentale de la sémantique générale, il donne à DIR(p) [ou à DIR'( $\left.\mathrm{p}^{\prime}\right)$ ] un statut d'acte de langage réussi. C'est-à-dire que c'est $L$ qui, rétroactivement, propose à $E$ d'actualiser son dire en une requête d'information [et respectivement en une requête d'action]. E cet instant de l'échange, le statut du dire de 
E est en balance. En effet, son locuteur peut soit ratifier la proposition (c'est le cas des séquences 1 et 2), soit l'invalider (c'est le cas des séquences 3 et 4). On a alors, au troisième tour de parole, une stabilisation du sens qu'a acquis le premier énoncé. Stabilisation et non pas fixation car ce statut est toujours négociable dans l'après conversationnel.

D'après nous, il n'est en aucun cas, plus justifié de dire, en tant qu'analyste, que $\mathrm{E}$ et $\mathrm{L}$ se sont compris dans l'une ou l'autre de ces séquences. De plus c'est un leurre que de penser que E lui-même, interrogé par après, pourra donner la clé et dire si c'est ce qu'il «avait voulu dire» car on aura affaire là à une reconstruction a posteriori. D'ailleurs peu importe de savoir si $\mathrm{L}$ a bien «découvert» ce que «voulait dire» E. Ce qui est central c'est qu'ils ont, au cours du développement de l'interlocution, fait émerger un sens de $E_{1}$ dont ils sont co-responsables. Parmi les quatre séquences, c'est la première qui a réellement été observée; le sens de $\mathrm{E}_{\mathrm{l}}$, alors stabilisé, a été recyclé plusieurs semaines plus tard par les deux mêmes interactants.

La justification théorique de cette analyse du phénomène qui survient en ce lieu d'intersubjectivité se trouve dans la convocation d'une relation logique entre satisfaction et succès de l'acte de langage assertif, littéral (pour séquence 1 et 3 ), ou de l'acte de langage directif, non littéral (pour séquence 2 et 4 ). Le détail de ce mécanisme logique qui est au cœur de ce que nous appelons la logique interlocutoire se trouve exposé longuement dans (Trognon et Brassac, 1992 ou dans Ghiglione et Trognon, 1993). L'idée fondamentale est que l'énoncé initial (contrairement à la théorie classique) n'a pas de statut illocutoire indépendamment de son traitement, au long de la conversation, par les deux interactants. Ce statut n'est pas le seul fait de son locuteur, il n'est pas non plus le seul fait de l'auditeur, il est élaboré, perlaboré à travers la négociation de sens par le couple d'interlocuteurs. Cet énoncé (1), dans cette conversation, ne possède pas un sens que le locuteur $E$ lui aurait attribué une fois pour toutes (que ce sens soit le sens littéral ou non); cet énoncé n'acquiert un sens que dans le jeu subtil de négociations (proposition d'actualisation, ratification ou invalidation), jeu dont les deux conversants sont les acteurs et qui conduit à la stabilisation, provisoire, d'un sens dont aucun des deux n'est propriétaire.

\subsection{CO-CONSTRUCTION DE SENS ET INTERCOMPRÉHENSION}

Co-responsables de cette stabilisation du statut interlocutoire des énoncés successivement produits, les acteurs de l'échange interlocutoire participent à la co-construction du sens de ces formes langagières qui tissent la conversation. Les énoncés proférés ne permettent pas la réalisation d'un acte de langage, celui qui correspondrait à ce l'on appelle couramment le sens littéral: tout le monde s'accorde à le dire et à le penser. Bien sûr, ils peuvent véhiculer des sens implicites, non littéraux. Mais ce qui est important, et ce sur quoi nous insistons avec vigueur, c'est que le locuteur ne contrôle pas ce qu'il peut advenir de ces implicites dans la suite de l'échange. Autrement dit, l'énoncé proféré est porteur d'un potentiel, non nécessairement dénombrable, de sens et c'est la dyade qui actualise, au cours de la conversation, un des éléments de ce potentiel (il peut s'agir d'un sens non prémédité par le locuteur initial). 
La conversation n'est pas un lieu de transfert d'un message, fût-il caché (au sens d'implicite), il est le lieu d'un façonnage des cognitions en présence. En reposant sur l'idée essentielle que le sens est co-construit, de façon processuelle et radicalement dialogique, cette façon de modéliser la conversation participe d'une perspective constructiviste (Brassac et Stewart, 1996). Point n'est besoin de postuler l'existence d'un sens préexistant et à l'expression et à la compréhension de la forme linguistique proférée en contexte. Il suffit d'accepter l'idée simple selon laquelle les interactants, immergés dans un potentiel subtilement impermanent de sens, le façonnent conjointement et processuellement et ainsi en font émerger un sens provisoire et toujours négociable.

Ainsi nous avancons l'idée que pour envisager scientifiquement les conduites humaines, il est nécessaire de les considérer comme des activités contextualisées dans des situations authentiques, interactionnelles; autrement dit, de les considérer comme des expressions incarnées de cognitions distribuées et situées. C'est typiquement ce que nous faisons lorsqu'en relation avec des ingénieurs, des rédacteurs ou des développeurs de produits ou de processus de production, nous envisageons l'activité de conception comme un engendrement radicalement situé et distribué d'idées, de processus de décisions et autres cognitions. La deuxième partie décrit un des projets que nous menons dans ce champ d'études.

\section{CONCEVOIR INTERACTIVEMENT UN DIDACTICIEL}

Nous travaillons actuellement sur un projet de développement d'un didacticiel portant sur la formation à la maintenance des systèmes automatisés (Champy-Remoussenard et Grégori, 1998; Grégori et al., 1998). Nous poursuivons alors trois objectifs. Le premier est pédagogique. Il consiste à développer des outils multimédias pour le domaine de la formation en général. Le deuxième est méthodologique. Il porte sur l'élaboration d'un atelier de génie multimédia pour la création de didacticiels. Enfin le troisième concerne la collaboration effective et efficace des équipes de développement, les unes relevant des sciences humaines, formant l'équipe pédagogique, d'autres des sciences pour l'ingénieur, ayant en charge la conception et la production du système d'information qui soutient le didacticiel, et la dernière, enfin, du monde de l'industrie ayant la responsabilité des spécifications logicielles et du développement technique du didacticiel.

Or, toutes ces structures de développement et de recherche, et donc tous les individus qui composent ces structures, doivent communiquer entre elles, doivent se comprendre, doivent travailler ensemble. Et, bien entendu, ce qui n'est pas toujours aisé à mettre en œuvre au sein d'une même discipline ne l'est pas plus dans un tel contexte interdisciplinaire. Non seulement les langages ne sont pas les mêmes, mais en outre les objectifs de travail sont eux aussi particuliers, ce qui est typique du cadre de la conception distribuée (Salaü, 1995). Nous avons déjà analysé ce type de situa- 
tion en considérant l'utilisateur comme acteur du processus de conception (Brassac et al., 1997; Grégori et al., 1996; Grosjean et al., 1997). Cette perspective s'attache à rendre compte théoriquement de la complexité du dispositif comme nous allons le voir.

\subsection{L'ANALYSE DES INTERACTIONS DANS LE PROJET: INTÉGRER L'UTILISATEUR}

La question de plus en plus prégnante en conception d'outils multimédias est de tenir compte de l'utilisation (Mallein, 1996; Norman, 1996; Rabardel, 1995). Mais comment réaliser une telle «conception centrée sur l'utilisateur»? Commençons par exposer rapidement les modèles de conception des didacticiels (Coutaz, 1995; Foisy, 1995; Mallein, 1996; Norman, 1996; Surcin et al., 1995) en y caractérisant la place de l'usager, avant de montrer ce qu'une psychologie de l'interaction peut proposer comme piste de développement.

\subsubsection{LES MODĖLES DE CONCEPTION}

Le modèle en $\mathrm{V}$, figure 1 à gauche, concerne les cas de conception pour lesquels les finalités du produit sont bien déterminées (Coutaz, 1995). Les besoins de l'utilisateur sont traduits en spécifications pédagogiques, dans un premier temps, puis logicielles. C'est la phase descendante. Cela aboutit à la réalisation d'un didacticiel que l'on va alors tester lors d'une phase ascendante, d'un point de vue logiciel d'abord, puis d'un point de vue pédagogique. Le didacticiel peut alors être produit en série et vendu.

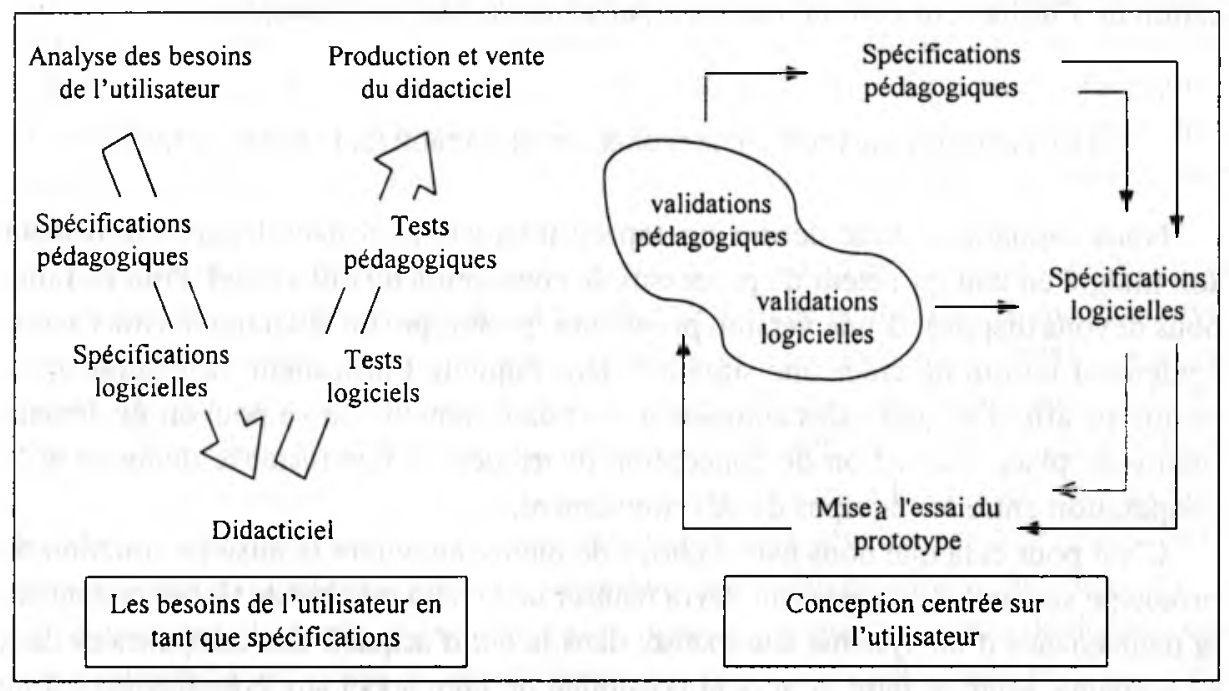

Figure 1. Les modèles de conception des didacticiels 
Lorsque les finalités du didacticiel sont moins bien connues, ce qui est le cas pour le projet dont nous discutons, on recourt plus généralement à une sorte de modèle en spirale (figure 1, à droite). L'incertitude sur le produit se traduit par le développement d'une version intermédiaire du produit, généralement appelée maquette ou prototype, et par la mise en test de cette version provisoire dans le but d'apprécier le développement tant d'un point de vue logiciel que pédagogique. Lorsque les analyses sont réalisées, une nouvelle itération est accomplie, si cela s'avère nécessaire.

Bien souvent ce modèle en spirale est présenté comme orienté utilisateur parce que ce dernier joue un rôle important lors de la phase de mise à l'essai de la version prototype du didacticiel. Toutefois, on peut s'interroger sur le fait que cela soit vraiment le cas. En effet, d'après nous, l'utilisateur a un rôle passif dans ce modèle. Il est une sorte de testeur d'interface, mais il ne participe pas véritablement à la conception elle-même du didacticiel. Il n'est pas un acteur de la conception. Dans ce modèle, chacun reste à sa place, les concepteurs conçoivent et les utilisateurs utilisent. Or si l'on veut, comme le prescrit Mallein (1996), se préoccuper de la notion d'usage à l'intérieur du processus de conception, certainement faut-il que l'utilisateur y soit présent de manière plus active.

Autrement dit, il nous semble que malgré les intentions affichées dans de nombreux travaux et présentations de développement multimédias, l'approche de conception reste majoritairement technocentrée (Rabardel, 1995), y compris dans l'utilisation d'un modèle en spirale. Technocentrée parce que l'utilisateur n'est pas doté d'une activité de développement. Si bien que la mise à l'essai du prototype aboutit à des activités de validations ou d'amendements alors que dans le cas d'une perspective anthropocentrée, on devrait aboutir à une activité de reconception du fait de la convocation de l'utilisateur comme nouveau partenaire/acteur de conception.

\subsubsection{CRÉER UN ESPACE POUR INTÉGRER L'UTILISATEUR EN TANT QU'ACTEUR}

Nous souhaitons donc définir un espace interactionnel dans lequel l'utilisateur soit intégré en tant qu'acteur du processus de conception du didacticiel. Pour ce faire, nous devons disposer d'une version provisoire (prototype) du didacticiel. Nous avons également besoin de créer une situation dans laquelle l'utilisateur va manipuler ce prototype afin d'acquérir des compétences et dans laquelle on va pouvoir également mettre en place une action de conception distribuée en favorisant le dialogue et la coopération entre les équipes de développement.

C'est pour cela que nous avons choisi de mettre en cuvre la mise en situation du prototype suivante. Un apprenant devra réaliser un certain nombre de tâches portant sur la maintenance d'un système automatisé, dans le but d'acquérir des compétences dans ce domaine. Pour ce faire, il aura la possibilité de faire appel aux connaissances d'un enseignant spécialiste de cette formation, qui, bien entendu, connaît les objectifs péda- 
gogiques du didacticiel ainsi que ces fonctionnalités, et aux connaissances d'un spécialiste du développement de ce didacticiel, ces deux personnes étant présentes à ses côtés.

Nous faisons l'hypothèse que dans une telle situation, des besoins, en termes de navigation ou d'assistance par exemple, seront exprimés par les acteurs en présence, que des solutions seront construites, que des procédures d'accomplissement des compétences émergeront. En d'autres termes, nous postulons que durant l'interaction, des cognitions vont émerger à partir du système mis en place. Et ce sont ces cognitions que nous souhaitons modéliser et implémenter dans le didacticiel.

En outre, remarquons bien que ce type de situation est une situation de conception distribuée, non seulement au sens «réseau» du terme, c'est-à-dire au sens où plusieurs expertises sont appelées à être exprimées en parallèle, mais aussi et surtout au sens constructiviste du terme, exposé plus haut, c'est-à-dire au sens où dans la situation décrite, les solutions émergeront en tant que cognitions co-construites, co-élaborées, négociées, c'est-à-dire distribuées sur les acteurs et les artefacts en présence.

En procédant de la sorte, il nous semble que nous mettons effectivement en place une conception centrée sur l'utilisateur puisque ce dernier n'a pas pour seul fonction de faire savoir aux concepteurs que leurs options de développement lui conviennent ou non. Certes, on attend de lui qu'il le fasse. Mais on attend également de lui qu'il soit acteur de la conception c'est-à-dire qu'il propose et discute les solutions émergentes. Cela est d'importance pour nous, chercheurs en sciences humaines, car notre fonction dans un processus de conception n'est pas de chercher à anticiper les besoins de l'utilisateur, ce que pourtant nous demande souvent et avec force nos partenaires de sciences pour l'ingénieur (Rogard et Vallery, 1993; Spérandio, 1993). Nous devons au contraire chercher à analyser l'utilisation des matériels en situation (Rabardel, 1995; Saint-Dizier, 1997). C'est-à-dire que nous devons analyser les cognitions distribuées et situées (Conein et Jacopin, 1994; Hutchins, 1995; Suchman, 1987) qui se nouent dans le cadre de l'utilisation du didacticiel par un apprenant, situation dans laquelle ce dernier a toute latitude pour discuter directement du développement de ce didacticiel.

Cette dernière revendication pourra peut-être choquer certains, tant il est habituel de considérer que l'utilisateur n'étant pas ingénieur, il ne peut avoir qu'une vue naïve, partielle, sans intérêt. À ceux-là nous rappellerons avec Rabardel (1995) que développer une perspective de conception anthropocentrique, c'est abandonner l'idée (technocentrique) que l'utilisateur et l'utilisation sont prédéfinis dans la machine. Linard (1996) rappelle de son côté qu'il est temps de rompre avec la croyance qu'il existe une sorte d'utilisateur-idéal pour lequel les concepteurs développent leur instruments, un peu comme il existe un locuteur-idéal dans la théorie généraiive de Chomsky.

Il nous reste bien sûr, et ce n'est pas le moindre de nos travaux, à démontrer que notre démarche est viable et qu'elle peut être efficace. Il nous reste bien sûr à démontrer que la notion d'usage dans sa globalité peut ếtre opérationnalisée et prise en 
compte dans un processus de conception d'un didacticiel. Mais la perspective ouverte nous semble si riche et si fondamentale, tant d'un point de vue industriel que d'un point de vue recherche, qu'il nous paraît indispensable de la creuser profondément dans le sens indiqué au long de cet article avant d'y renoncer. En tout cas sous cette forme. Nous conclurons donc en prenant à notre compte le mot d'ordre de Mallein (1996): «il faut banaliser l'usage et non pas l'imposer». Gageons que considérer l'utilisateur comme partenaire-acteur de l'activité de conception, et non pas comme un simple testeur, y contribuera.

\section{CONCLUSION}

L'objectif de ce travail de psychologue de l'interaction n'était pas de montrer le bien-fondé de la mise au service de l'analyse du discours au profit des sciences pour l'ingénieur et des sciences de la didactique (actuellement friands d'outils multimédia pédagogiques). Loin de nous l'idée qu'une recherche fondamentale doit se distinguer de son homologue qui ne serait qu'applicative. Au contraire, c'est d'une recherche engagée dans l'action qu'il s'agit là. Recherche qui se nourrit en retour des richesses contingentes de l'expérimentation (au sens vygotskien du terme), qui se nourrit des aléas de la mise en situation des acteurs concepteurs.

Ainsi, cette dernière est-elle productrice d'événements imprévisibles et inattendus pour les conversationnalistes que nous sommes. Sujets humains plongés en situation d'usage d'un logiciel en cours d'élaboration, les acteurs manipulent, hors le langage, une multitude d'objets (souris, clavier, figures, fenêtres, etc.) qui sont autant d'intermédiaires dans le processus entre eux (les deux acteurs) et entre eux et le monde. Ces intermédiaires, de plus en plus étudiés (Jeantet et al., 1994; Jeantet, 1998, par exemple), jouent un rôle considérable dans le processus de conception. L'intégration de ces objets médiateurs produit, par exemple, un renouvellement significatif de notre vision de l'interaction langagière tournée initialement plutôt vers les aspects plus strictement discursifs. C'est de cette extension qu'il s'agit lorsque nous utilisons la locution «cognition distribuée et située».

\section{BIBLIOGRAPHIE}

B rass ac, Ch. (1994), Speech acts and conversational sequencing, Pragmatics and cognition 2(1), 191-205. [1994a].

B ras s a c, Ch. (1994), Actes de langage et enchaînement conversationnel, Cahiers d'épistémologie, $n^{0}$ 9401, Université du Québec à Montréal. [1994b].

B rassac, Ch. (1992), Analyse de conversations et théorie des actes de langage, Cahiers de Linguistique Française 13: 62-76. 
Brassac, Ch., Grosjean, S., Grégori, N. (1997), La psychologie interactionniste comme outil d'analyse des dialogues de conception, Joumée scientifique de l'ARC, 8 décembre 1997.

Brassac, Ch., Stewart, J. (1996), Le sens dans les processus interlocutoires, un observé ou un co-construit? Cinquièmes Journées de Rochebrune „Du social au collectif ”, 29 janvier 3 février 1996.

Champy-Re moussen ard, P., Grégori, N. (1998), Hypermédias éducatifs: conception et précautions d'usage. 4ème colloque Hypermédias et Apprentissages, Poitiers, 15-17 octobre 1998.

Conein, B., Jacopin, E. (1994), Action située et cognition: le savoir en place, Sociologie du travail, XXXVII 4/94, 475-499.

Coutaz, J. (1995), Interaction homme-machine: points d'ancrage entre ergonomie et génie logiciel, in: J. Caelen et Kh. Zreick (éds), Le communicationnel pour concevoir. Paris, Europia, 245-254.

Fo is y, L. (1995). Didacticiels multimédias, L'informateur, vol. $3 / 2$.

Ghiglione, R., Trognon A. (1993), Où va la pragmatique? De la pragmatique à la psychologie sociale. Grenoble, Presses Universitaires de Grenoble.

Grég ori, N., Grosje an, S., B rass ac, Ch. (1996), L'ontologie conjointement construite des objets en conception distribuée. Sixième colloque de l'ARC, Lille, 10-12 décembre 1996.

Grégori, N., Re mous senard, P., B ras s ac, Ch., Mayer, F. (1998), A design method for educational multimedia software, Proceedings of the IFAC-INCOM'98, Nancy-Metz, June 24-26, 1998, vol. 3, 237-242.

Grosjean, S., Brassac, Ch. (1997), L'émergence de l'objet: de l'objet cognitif à l'objet social. Actes de 0IDESIGN'97, Les objets en conception, Théoule-sur-mer, 24-26 septembre 1997, 91-103.

Hutchins, E. (1995), Cognition in the wild. Cambridge, MIT Press.

J e antet, A. (1998), Les objets intermédiaires dans la conception. Eléments pour une sociologie des processus de conception, Sociologie du traviil XL, 3/98, 291-316.

Jeantet, A., Tiger, H., Vinck, D., Tichkiewitch, S. (1994), La coordination par les objets dans les équipes intégrées de conception de produir, in: Conception et coopération, Paris, Europia: 87-100.

Linard, M. (1996), Des machines et des hommes, Paris, L'Harmattan.

Malle in, Ph. (1996), La conception assistée par l'usage, une extension du champ d'application de la conception à l'écoute du marché. Communication aux assises de la recherche en qualité, Versailles, 10-11 décembre 1996.

Norman, D. (1996), Grandeur et misère de la technologie, La Recherche, 285, 22-25.

$\mathrm{R}$ abardel, P. (1995), Les hommes et les technologies: approche cognitive des instruments contemporains, Paris, A. Colin.

Rogard, V., Vallery, G. (1993), L'ergonomie cognitive dans le processus d'informatisation des activités tertiaires, in: J.C. Spérandio (éd.), L'ergonomie dans la conception des projets informatiques, Toulouse, Octarès.

Saint-Dizier de Almeida, V. (1997), Modélisation d'une assistance interactive pour améliorer l'accessibilité d'un logiciel, Sciences et techniques éducatives, vol. 4/1, 13-39.

Sal aü, I. (1995), La conception distribuée: Théorie et méthodologie. Thèse de doctorat en automatique, Nancy, Université Henri Poincaré.

Searle, J. R., Vanderveken, D. (1985), Foundations of illocutionary logic, Cambridge, CUP. 
Suchman, L. (1987), Plans and situated actions, the problem of human-machine communication, Cambridge, CUP.

Surcin, S., Choppy, Ch., Séré, M.G. (1995), Analyse didactique d'un cours de génie logiciel basé sur l'approche orientée objets, Sciences et techniques éducatives, vol. 2/3, 265-286.

Trognon, A., Brassac, Ch. (1995), Formalizing the theory of intentionality, Journal of Pragmatics 23: 555-562.

Trognon, A., Brassac, Ch. (1992), L'enchainement conversationnel, Cahiers de Linguistique Française 13: 76-107.

Vanderveken, D. (1988), Les actes de discours, Bruxelles, Mardaga. 